\title{
Dust Composition in the Low Density Medium Around Spica
}

\author{
F. Zagury, A. Jones, and F. Boulanger \\ Institut d'Astrophysique Spatiale Université Paris-Sud, Bâtiment 121, \\ F-91405 Orsay Cedex, France
}

\begin{abstract}
Dust grains in low density gas are subjected to sputtering and shattering in fast supernovae shocks. Although there has been extensive modelling of the dust destruction, still little is known about the grains which survive. Jones et al. (1996) have modeled the effect of the destruction processes on the size distribution of grains. They predict that fast $\mathrm{J}$ shocks are efficient in grinding large dust grains into smaller particles. We present observations of the dust emission around the nearby star Spica. Comparison of the observations to the infrared emission of 'standard' cirrus suggest that the abundance of very small grains is significantly enhanced.
\end{abstract}

\section{Introduction}

Depletion studies of the grain forming elements ( $\mathrm{C}, \mathrm{Mg}, \mathrm{Fe}, \mathrm{Si}, \mathrm{O}$, etc...) show that interstellar grains are partly destroyed in the low density phases of the interstellar medium. We present a first study of the infrared emission around Spica. Spica, a B1 III-IV star, is surrounded by a vast $18^{\circ}$ in extent HII region of low density, $n \sim 0.5 \mathrm{~cm}^{-3}$ (Reynolds 1985, York and Kinahan 1978). The presence of the star enhances the dust infrared emission, which becomes comparable to that of denser cirrus and can be separated from the background cirrus emission.

\section{Spica Situation Relative to the Local Bubble}

Spica (Galactic coordinates $l=316.11, b=50.84$ ) is at $80 p c$ from the Sun, in the direction of the Scorpius Centaurus association. It is probably located close on the outer side of the cavity blown by the association, defined by Loop I: the column density of gas $\left(N_{H} \sim 10^{19} \mathrm{~cm}^{-2}\right)$ towards the star is lower by an order of magnitude than that measured for stars inside the bubble (Centurion and Vladilo 1991). On the other hand its distance from the Sun puts Spica near the edge and outside the Local Bubble. In figure 1 the HI emission to the East of Spica might be the HI shell around Loop I which would clearly prove that Spica is outside the Scorpius Centaurus Bubble. The low NaI/CaII ratio on the line of sight toward Spica suggests that the region has been crossed by shock waves (Centurion and Vladilo 1991). 


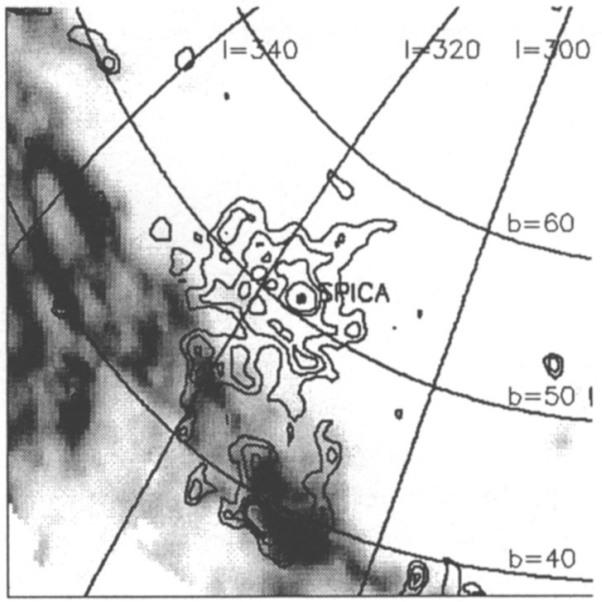

Fig. 1. HI EMISSION AROUND SPICA

VLSR ranges from $-25 \mathrm{~km} / \mathrm{s}$ to $25 \mathrm{~km} / \mathrm{s}$. Bright $\mathrm{HI}$ emission appears dark on the image. Contours are $100 \mu \mathrm{m}$ DIRBE emission from which the correlated part of the $\mathrm{HI}$ gas has been subtracted. The contour values are : $0.5,0.8,1.1,1.4 \mathrm{MJy} / \mathrm{sr}$. Some $\mathrm{HI}$ and $100 \mu \mathrm{m}$ emission are still correlated. This might be due to the presence of molecular gas in the brightest HI features.

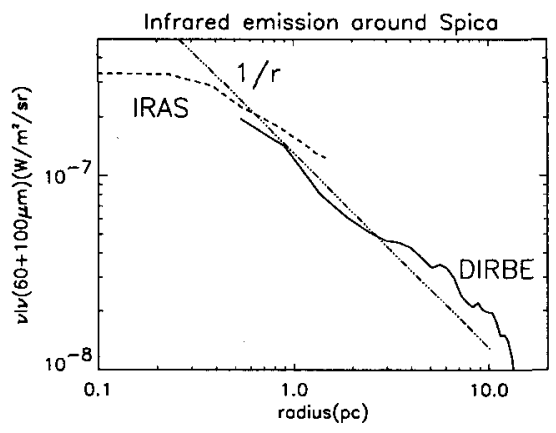

Fig. 2. Sum of $\nu I_{\nu}$ emission from $60 \mu \mathrm{m}$ to $100 \mu \mathrm{m}$ for IRAS et DIRBE versus distance to the star. A $1 / r$ slope is compared to infrared emission.

\section{Infrared Emission of the HII Region Around Spica}

In figure 1 superposed on the HI map are the $100 \mu \mathrm{m}$ emission contours, after subtraction of that part correlated with the HI emission. The infrared emission associated with the $\mathrm{HI}$ gas was estimated by scaling the spatial distribution of the HI emission taken from the Leiden-Dwingeloo survey (Hartmann and Burton 1995) with the average IR/HI ratio measured at high Galactic latitude (Boulanger et al. 1996). The residual infrared emission is centered 
on Spica and comes from the HII region around the star. In figure 2 the integrated emission from 60 to $100 \mu \mathrm{m}, \nu I_{\nu}$, is plotted versus the distance (in pc) to the star for both IRASand DIRBE data. It follows a $1 / \mathrm{r}$ law, expected for the emission from an homogeneous medium heated by a central star. This attests that the infrared emission we observe does come from Spica's HII region.

\section{Modelling the Infrared Emission}

Desert et al. (1990) have modelled the emission of infrared cirrus. This model includes 3 components : big grains, very small grains (VSG) and Polycyclic Aromatic Hydrocarbons (PAH). Big grains have a black body like emission and are responsible for part of the $60 \mu \mathrm{m}$ emission and all the emission above $100 \mu \mathrm{m}$. VSGs are transiently heated particles which radiate between $25 \mu \mathrm{m}$ and $60 \mu \mathrm{m}$. PAHs make the infrared emission at shorter wavelengths. We apply this model to Spica's HII region. The star's luminosity determines the radiation field and is that of a black body at a temperature of $27000 \mathrm{~K}$ (from Spica's spectral type) whose intensity can be found by the visual magnitude of Spica. We assume the medium to be homogeneous with $\mathrm{n}=0.5 \mathrm{~cm}^{-3}$ derived from $\mathrm{H} \alpha$ observations.
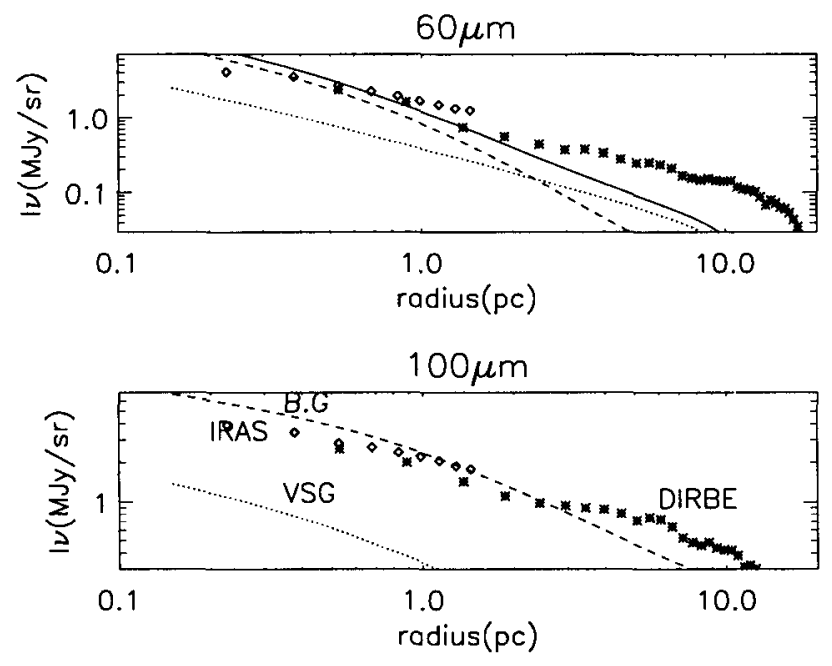

Fig. 3. MODEL OF THE INFRARED EMISSION AROUND SPICA

Dust emission at 60 and $100 \mu \mathrm{m}$ for a medium of density $0.5 \mathrm{~cm}^{-3}$ in Spica's radiation field is plotted versus the distance from the star. For comparison DIRBE and IRAS emission are also plotted. 


\section{Discussion}

In figure 3 the model and the data are compared. While big grains can account for the $100 \mu \mathrm{m}$ emission, the $60 \mu \mathrm{m}$ predicted emission is too low at $r \geq 4 \mathrm{pc}$. In figure 4 we present DIRBE spectrum $5 \mathrm{pc}$ from the star and the relative contributions of PAH, VSG and big grains as set by the model. These figures convincingly demonstrate that it is necessary to enhance the VSG emission by a factor of 2 to 3 to reproduce the observed spectrum. Since Spica is located in a low density region of the interstellar medium at the edge of the Loop I Bubble it is tempting to associate the enhanced abundance with a shock as predicted by Jones et al (1996) .

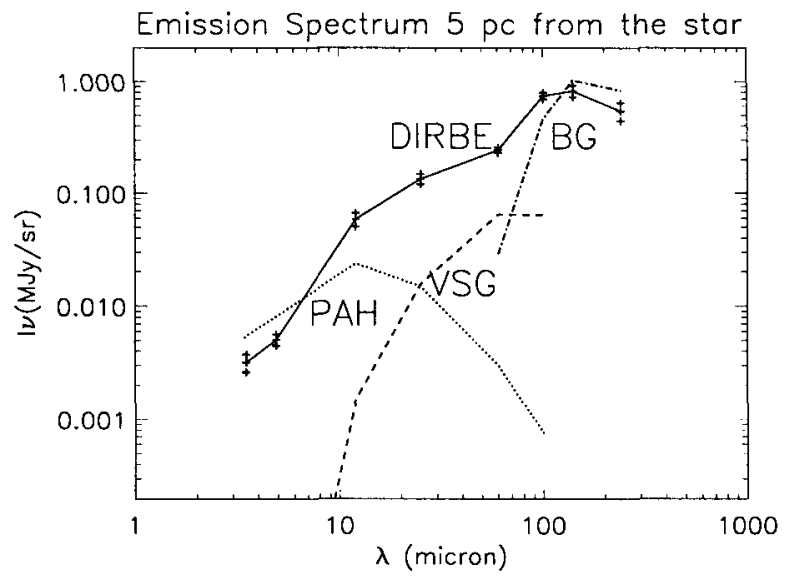

Fig. 4. DIRBE emission spectrum, 5 pc from the star, is compared to that expected from an average cirrus of the Galaxy (our model).

\section{References}

Boulanger, F., et al. (1996): A\&A 312, 256

Centurion, M., Vladilo, G. (1991): ApJ 372, 494

Desert, F. X., Boulanger, F., Puget, J.L.(1990): A\&A 237, 215

Hartmann, D., Burton, W.B (1995): Atlas of Galactic HI emission. Cambridge University Press

Jones, A.P., Tielens, A.G.G.M., Hollenbach, D.J. (1996): ApJ 469, 740

Reynolds, R.J. (1985): AJ 90, 92

York, D.G., Kinahan, B.F. (1979): ApJ 228, 127 